\title{
Single-particle subband spectroscopy in a parabolic quantum well via transport experiments
}

\author{
K. Ensslin and A. Wixforth \\ Sektion Physik, Universität München, Geschwister-Scholl Platz 1, 8000 München 22, Germany \\ M. Sundaram, P. F. Hopkins, J. H. English, and A. C. Gossard \\ Materials Department, University of California, Santa Barbara, California 93106
}

(Received 4 May 1992)

\begin{abstract}
The transport properties of a parabolic quantum well are investigated at low temperatures 0.5 $\mathrm{K}<T<4 \mathrm{~K}$. A front-gate voltage is used to tune the carrier density and hence the subband structure over a wide range. Various orientations of a magnetic field $B$ with respect to the normal of the sample $0^{\circ} \leq \alpha \leq 90^{\circ}$ are investigated. For $\alpha=0^{\circ}$, where $B$ is oriented along the surface normal of the sample, quantum Hall states can be suppressed and recovered depending on the carrier density of the sample. This effect is explained quantitatively by a self-consistent calculation that considers the interplay of occupied Landau levels belonging to different subbands. A suppressed quantum Hall state can also be recovered via a tilted magnetic field. The resonant-subband-Landau-level coupling leads to a repulsion of the levels and, therefore, to the creation of an energy gap. For $T \leq 0.5 \mathrm{~K}$ the spin splitting of the Landau levels appears in the magnetoresistance. In the regime of carrier densities where the quantum Hall plateau corresponding to filling factor $v=4$ is suppressed, a double minimum structure for $v=3$ is observed, reflecting the different exchange enhancement of the spin splitting of the two lowest subbands. For magnetic fields oriented in the plane of the sample, a band structure arises that leads to an anisotropic conductivity for current flow perpendicular and parallel to the magnetic-field orientation.
\end{abstract}

\section{INTRODUCTION}

Subband spectroscopy in low-dimensional semiconductors is usually accomplished with optical experiments. ${ }^{1}$ Transitions from occupied to empty states in a twodimensional electron (2DEG) have been studied by Raman spectroscopy ${ }^{2}$ and by far-infrared (FIR) spectroscopy in a strip-line configuration. ${ }^{3}$ In tilted magnetic fields the resonant-subband-Landau-level coupling (RSLC) was used $^{4-9}$ to determine the subband separations from the coupling of the cyclotron resonance and the intersubband resonance. These powerful experimental techniques suffer from the fact that the energies they determine are influenced by collective effects. ${ }^{10,11}$ This is especially true in parabolic quantum wells where there exists the unique situation that the RSLC indeed occurs between the plasma frequency and the respective Landau levels. This has been shown theoretically ${ }^{12}$ and confirmed experimentally. 9,13

In this paper we present a series of magnetotransport experiments that allow the direct determination of the single-particle subband separations in a parabolic quantum well. For magnetic fields oriented perpendicular to the sample a series of equidistant Landau levels develops on each subband. The carrier density and therefore the subband structure can be controlled reproducibly via a front-gate voltage. For a given set of parameters two Landau levels belonging to different subbands can be degenerate at the Fermi energy. In this case the corresponding quantum Hall state is suppressed. It can be recovered by lifting the degeneracy of these Landau levels via a suitable gate voltage. If the magnetic field is tilted with respect to the normal of the sample these Landau levels repel each other and the degeneracy is also lifted. In both cases the corresponding quantum Hall state is recovered. These experiments depend sensitively on the subband structure and allow a detailed comparison of experiment and calculation. At low temperatures, $T \leq 0.5$ $\mathbf{K}$, the Zeeman splitting of the Landau levels is resolved. An especially interesting situation occurs when the quantum Hall state for filling factor $v=4$ is suppressed, while a double minimum structure in the vicinity of $v=3$ is observed in the magnetoresistance. This behavior is explained by the subband dependent exchange enhancement, that leads to an effective charge transfer between the two subbands. An anisotropic band structure arises for magnetic fields oriented in the plane of the sample. This is caused by the fact that the effective mass as well as the scattering time depend on the direction of the field within the sample plane. This is strongly apparent in the conductivity for current flow parallel and perpendicular to the magnetic-field orientation. The features in the magnetoresistance arising from the magnetic depopulation of subbands are much more pronounced for current flow perpendicular to the magnetic-field orientation compared to the parallel orientation. This independent method of determining the single-particle subband energies in a transport experiment gives results that are in good agreement with the experiments described before.

The paper is arranged as follows: Section II describes in detail the self-consistent confining potential and subband occupation of the sample, Sec. III presents magnetotransport experiments for $\alpha=0$, where $\alpha$ is the angle between the normal of the sample and the magnetic-field orientation. In this case transport is dominated by the formation of Landau levels. ${ }^{14}$ Section IV shows that the spin splitting of Landau levels dramatically influences the 
magnetoresistance. Section $\mathrm{V}$ focuses on the tilted field case $0 \leq \alpha \leq 60^{\circ}$ with emphasis on the manifestation of resonant-subband-Landau-level coupling. ${ }^{15}$ Section VI contains results from the in-plane field case $\alpha=90^{\circ}$, where the magnetic depopulation of the subbands dominates the transport properties. Section VII summarizes the results.

\section{CONFINING POTENTIAL AND OCCUPATION OF SUBBANDS}

The GaAs- $\mathrm{Al}_{x} \mathrm{Ga}_{1-x}$ As parabolic quantum well (PQW) is grown by molecular-beam epitaxy. On top of the semi-insulating substrate there is a $400-\mathrm{nm}$ GaAs buffer layer, followed by $200-\mathrm{nm} \mathrm{Al}_{0.3} \mathrm{Ga}_{0.7} \mathrm{As}, 16-\mathrm{nm}$ $\mathrm{Al}_{0.3} \mathrm{Ga}_{0.7} \mathrm{As}$ with $\mathrm{Si}$ doping $\left(N_{D}=2.5 \times 10^{17} \mathrm{~cm}^{-3}\right), 20-$ $\mathrm{nm} \mathrm{Al}_{0.3} \mathrm{Ga}_{0.7} \mathrm{As}$ spacer, the $75-\mathrm{nm}$-wide parabolic well with varying $0<x<0.1$. The $\mathrm{Al}$ content $x$ is varied through a defined sequence of shutter openings and closings producing a $2-\mathrm{nm}$ period superlattice to achieve a parabolic variation of the conduction band. ${ }^{16}$ The cap is formed by a $20-\mathrm{nm} \mathrm{Al}_{0.3} \mathrm{Ga}_{0.7} \mathrm{As}$ spacer and a $101-\mathrm{nm} \mathrm{Si}$ doped $\mathrm{Al}_{0.3} \mathrm{Ga}_{0.7} \mathrm{As}\left(N_{D}=2.5 \times 10^{17} \mathrm{~cm}^{-3}\right)$ layer. The well is designed to have a symmetric electron distribution within the well. At $T=4.2 \mathrm{~K}$ and $V_{g}=0$ the total carrier density is $N_{s}=5 \times 10^{11} \mathrm{~cm}^{-2}$ and the mobility of the electron gas in the well is $\mu=100.000 \mathrm{~cm}^{2} / \mathrm{V} \mathrm{s}$. The mesa structure consists of two Hall bars that are oriented perpendicular to each other. The width of the current path is $50 \mu \mathrm{m}$ and the spacing between the voltage probes is $150 \mu \mathrm{m}$. Ohmic contacts are made by annealing $\mathrm{AuGe} / \mathrm{Ni}$ and a front gate $(\mathrm{Ti} / \mathrm{Au})$ is evaporated onto the sample. This allows us to tune the carrier density in the parabolic well and with it the width of the electron layer and the number of occupied subbands. ${ }^{17}$ The lowfrequency $(v \approx 30-\mathrm{Hz})$ transport experiments are performed in a superconducting magnet $(0-12 \mathrm{~T})$ and the samples are cooled by $\mathrm{He}$ exchange gas at temperatures $0.5 \mathrm{~K}<T<4.2 \mathrm{~K}$. For this section the magnetic field is oriented perpendicular to the plane of the electron gas. The samples are cooled down in the dark.

Figure 1 presents the results of a self-consistent calculation for the conduction-band edge, the occupied subbands, and the wave functions of the investigated sample. The carrier density is $N_{s}=5 \times 10^{11} \mathrm{~cm}^{-2}$. The surface of the sample is on the left-hand side of the figure. The inset shows the region of the potential where the electrons actually reside. The symmetry of the wave functions indicates that the hard-wall potential on the right-hand side of the potential does not affect the electron density or the energy levels strongly. The flatness of the potential over a wide region (almost $40 \mathrm{~nm}$ ) as well as the total carrier distribution shows the quasi-3D nature of the filled parabolic potential. According to the generalized Kohn's theorem $^{12}$ an optical intersubband spectroscopy experiment will always detect the plasma frequency of the electron system $8,9,13$ independent of the electron-electron interaction and therefore the number of electrons. A transport experiment will rely on the single-particle energy levels and therefore depend on the energies and wave

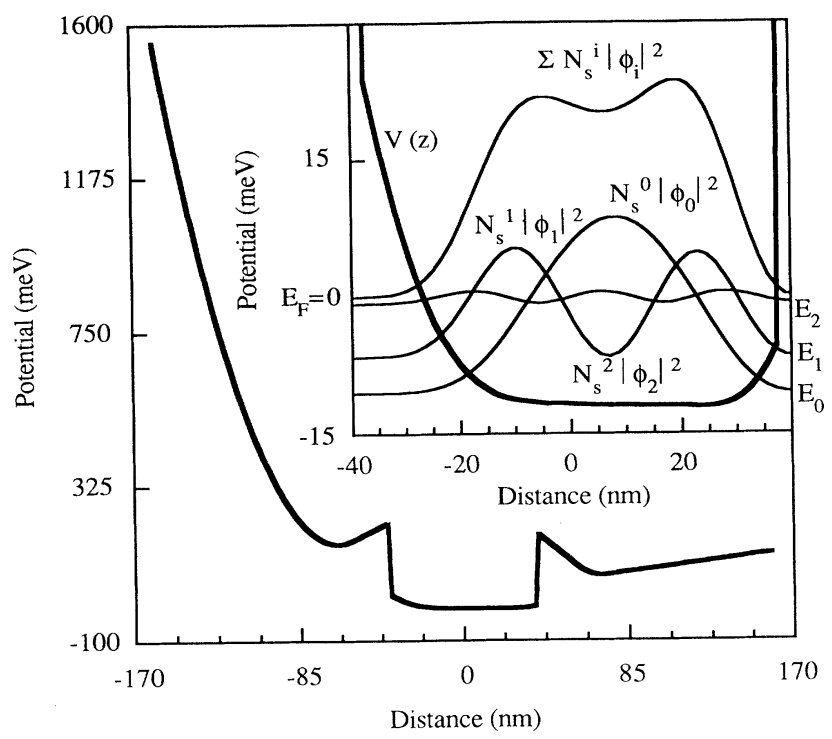

FIG. 1. Self-consistent calculation of the potential of the parabolic well with the surface of the sample (front gate) on the left-hand side of the figure. The inset is an enlarged scale of the potential well and the wave functions weighted by their corresponding subband carrier densities.

\section{functions as depicted in Fig. 1.}

Figure 2 presents experimental results on the mobility of the electrons as a function of carrier density. The evaluation of the mobility from the magnetoresistance is usually not a straightforward procedure for systems with more than one occupied subband. ${ }^{18-25}$ In cyclotron resonance ${ }^{26,27}$ the scattering time of a subband can directly be deduced from the linewidth of the respective resonance. In a transport experiment a model has to be used to extract scattering times of various subbands. ${ }^{18,20,23}$ Van

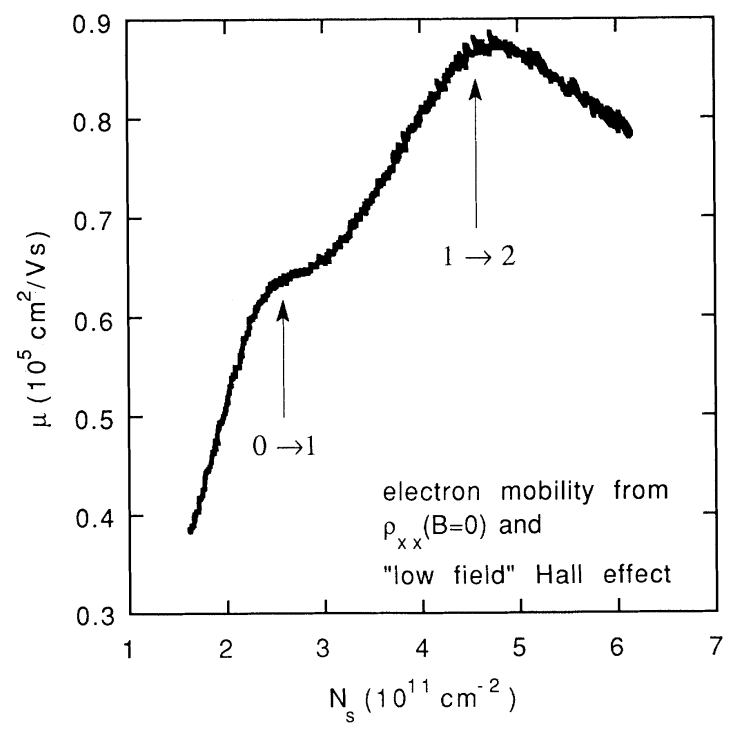

FIG. 2. Mobility at $T=4.2 \mathrm{~K}$ as a function of carrier density tuned by a gate voltage. The arrows indicate where the $E_{1}$ and $E_{2}$ subbands become populated and consequently a channel for intersubband scattering opens up. 
Houten et al. (Ref. 24) showed that for a high-mobility 2DEG with two occupied subbands a positive magnetoresistance $\Delta R$ occurs, with $\Delta R / R \propto\left(\mu_{0}-\mu_{1}\right)^{2}, \mu_{i}$ being the Drude mobility of the carriers in subband $i$. The measurement of the magnetoresistance $\rho_{x x}$ on the presented PQW reveals a flat behavior, i.e., $\rho_{x x}$ does not depend on $B$ for very small magnetic fields $B<0.2 \mathrm{~T}$ and for all temperatures and gate voltages investigated. We thus assume that the scattering time in all occupied subbands is nearly the same and we can therefore simplify the analysis of the mobility as presented in Fig. 2. The Hall effect $\rho_{x y}$ has been measured for a low magnetic field as a function of gate voltage. A linear relation between the carrier density $N_{s}$ and the gate voltage $V_{g}$ is obtained in agreement with the evaluation of the $1 / B$ periodicity of the Shubnikov-de Haas oscillations at high magnetic fields. The data in Fig. 2 clearly show a nonmonotonic behavior for the mobility $\mu$ as a function of carrier density. For two-dimensional electron gases with only one occupied subband a power-law dependence of $\mu\left(N_{s}\right)$ has been observed ${ }^{28}$ and explained by ionized impurity scattering. The same is observed in our sample below $N_{s}=2.5 \times 10^{11} \mathrm{~cm}^{-2}$. For higher carrier densities a second subband becomes populated which opens up an additional scattering channel, i.e., intersubband scattering occurs. This explains the structure in Fig. 2 marked by the first arrow. Similar effects have been observed in GaAs heterostructures (Refs. 18 and 24). A much more pronounced structure however occurs in our data around $N_{s}=4.5 \times 10^{11} \mathrm{~cm}^{-2}$, where the mobility has a maximum and then drops for increasing $N_{s}$. At this value of the carrier density a third subband becomes populated leading to a further increased intersubband scattering rate. The wave-function overlap which determines the strength of intersubband scattering is especially large between the lowest (denoted by quantum number $i=0$ ) and the third ( $i=2$ ) subband. This explains the drop of $\mu$ as a function of increasing $N_{s}$ as soon as the third subband becomes occupied. This sets the limits for the experimentally accessible parameters of this parabolic quantum well.

\section{SUPPRESSION AND RECOVERY OF QUANTUM HALL STATES}

We will now concentrate on the transport properties of the electron gas under high magnetic fields $B$ that are oriented perpendicular to the surface of the sample. Figure 3 presents data for the Hall resistance $\rho_{x y}$ as a function of magnetic field at a low temperature $T=0.45 \mathrm{~K}$. Three specific values of $N_{s}$ are selected. The quantum Hall plateau for filling factor $v=2\left(v=h N_{s} / e B\right)$ is very wide and almost independent of the value of $N_{s}$. The well-defined plateau for $v=3$ at $N_{s}=4.6 \times 10^{11} \mathrm{~cm}^{-2}$ becomes weaker and finally vanishes for decreasing $N_{s}$. This is explained by the decrease of the mobility which leads to an overlap of the broadened spin-split Landau levels. A more detailed discussion on the intriguing behavior of the spin states follows in Sec. IV. The interesting filling factor in this figure is however $v=4$. The corresponding quantum Hall plateau is well developed for $N_{s}=4.6 \times 10^{11} \mathrm{~cm}^{-2}$, completely suppressed for an inter-

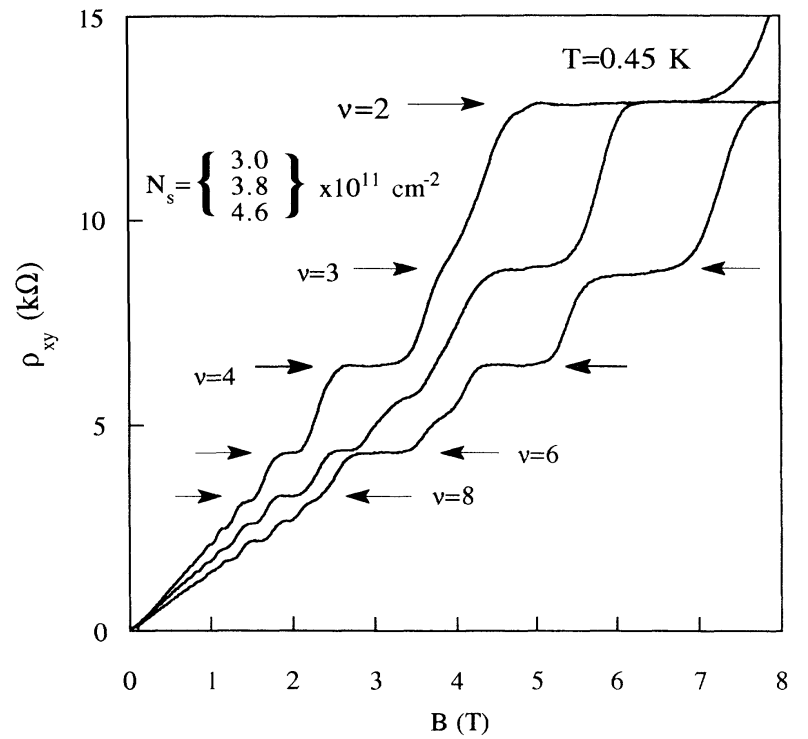

FIG. 3. Hall resistance curves for three different carrier densities measured at low temperatures $T=0.45 \mathrm{~K}$. The quantum Hall plateau corresponding to $v=4$ vanishes and reappears in this range of $N_{s}$.

mediate value of $N_{s}=3.8 \times 10^{11} \mathrm{~cm}^{-2}$, and entirely recovered for $N_{s}=3.0 \times 10^{11} \mathrm{~cm}^{-2}$. A similar behavior is observed for filling factors $v=8$ and 12 , whereas the suppression of quantum Hall plateaus for $v=2$ and 6 is never observed experimentally. The magnetoresistance $\rho_{x x}$ is always correlated with the Hall resistance $\rho_{x y}$ : a pronounced minimum in $\rho_{x x}$ leads to a quantum Hall plateau in $\rho_{x y}$, a suppressed minimum in $\rho_{x x}$ consequently results in a suppressed quantum Hall plateau in $\rho_{x y}$. We will concentrate in the following discussion on the behavior of $\rho_{x x}$, because it can more easily be compared with a theoretical calculation. We will neglect spin splitting in this section, because it is not resolved in the presented measurement.

Figure 4(a) presents a series of magnetoresistance measurements at $T=2 \mathrm{~K}$. The curves are vertically offset for clarity. The positions of filling factor $v=4$ are marked by arrows. In this range of carrier densities two subbands are occupied (see Fig. 1) at $B=0$, so that a minimum in $\rho_{x x}$ does not necessarily coincide with the position of an integer filling factor. For intermediate carrier densities there is actually a maximum in Fig. 4(a) for $v=4$. In the following we denote the total filling factor of the PQW by $v$; the filling factor of the subband $i$ is given by $v^{i}=h N_{s}^{i} / e B, N_{s}^{i}$ being the carrier density of subband $i$. In high magnetic fields carriers may be redistributed among the different subbands but the total carrier density $N_{s}$ does not depend on $B$. To understand the data as presented in Fig. 4(a), we solved self-consistently Schrödinger's and Poisson's equation in the presence of a magnetic field. All parameters for the calculation are given by the structure design. The Landau levels are modeled by a Gaussian density of $\operatorname{states}^{29}$ (DOS) with full width at half maximum $\Gamma[\mathrm{meV}]=0.5 \times(B[T])^{1 / 2}$. These parameters described reasonably well previous 


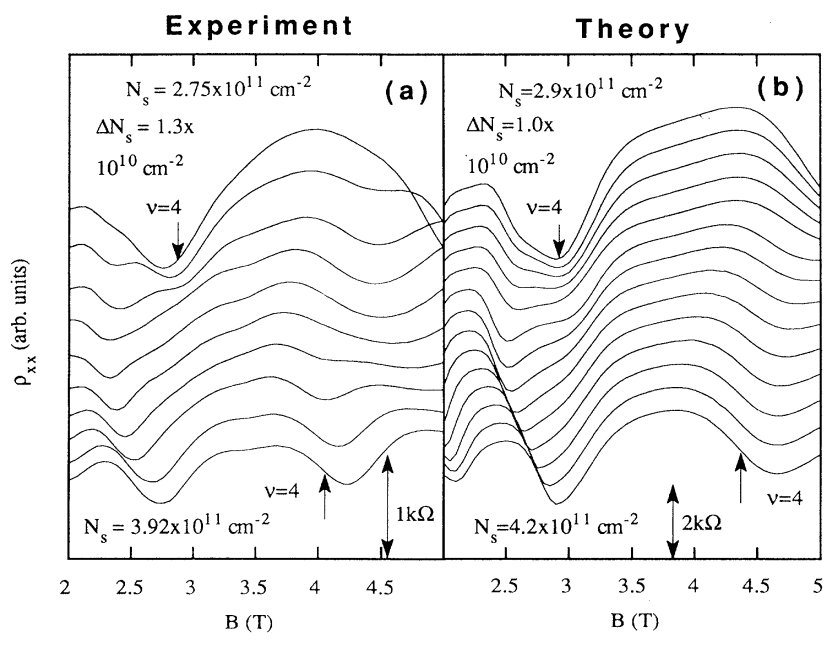

FIG. 4. (a) Experimental magnetoresistance traces for a series of carrier densities at $T=2 \mathrm{~K}$. The arrows indicate the position of filling factor $v=4$. The curves are vertically offset for clarity. The vertical scale is indicated by an arrow. (b) Calculated magnetoresistance traces as described in the text. Note the similarity to the data of $4(a)$.

magnetocapacitance measurements ${ }^{30,31}$ on samples with similar mobilities. Consequently, there are no adjustable parameters. We did not take into account complications such as spin splitting of the Landau levels, a constantbackground DOS (Ref. 32) or a filling-factor-dependent DOS. $^{30}$ The results are not qualitatively changed by a more refined model of the DOS. From the DOS we calculate the magnetic-field-dependent conductivity $\sigma_{x x}(B) .{ }^{29}$ Since the Hall resistance behaves almost classically in the interesting regime due to the suppression of the Hall plateaus it is reasonable to assume $\sigma_{x y}=-e N s / B$ for the Hall conductivity. This allows us to calculate $\rho_{x x}(B)$ as presented in Fig. $4(\mathrm{~b})$. The overall agreement between experiments [Fig. 4(a)] and theory [Fig. 4(b)] is very good, especially the position of $v=4$ with respect to the minima and maxima of $\rho_{x x}$. The same method of calculating the magnetoresistance but using a magnetic-field-independent subband structure was applied by Gobsch, Schulze, and Paasch ${ }^{33}$ to explain the data of Guldner et al ${ }^{34}$ on heterostructures with two occupied subbands.

An interesting feature in Figs. 4(a) and 4(b) is the double minimum structure in the range $2.5 \mathrm{~T}<B<3 \mathrm{~T}$. The top part of Fig. 5 presents a calculated magnetoresistance trace for $N_{s}=3.2 \times 10^{11} \mathrm{~cm}^{-2}$. The three figures of the lower part show the DOS for three magnetic fields that correspond to the regions on the $\rho_{x x}$ trace as marked by the arrows. Note that the DOS of each subband as well as the total DOS are plotted below and above the Fermi energy $E_{F}$. The Landau levels will be denoted in the following by $|i, n\rangle$, where $i$ is the subband index and $n$ the Landau-level index. In going from $B=2.4 \mathrm{~T}$ to $B=2.9$ T the state $|0,1\rangle$ (dashed line) approaches $|1,0\rangle$ (dotted line) because, $|0,1\rangle$ shifts up from the bottom of $i=0$ subband $E_{0}$ with $\frac{3}{2} \hbar \omega_{c}$ while $|1,0\rangle$ moves up by $\frac{1}{2} \hbar \omega_{c}$ from $E_{1}$. For an intermediate field $B=2.65 \mathrm{~T}$ the $|1,0\rangle$ state is just in between the $|0,1\rangle$ and $|0,2\rangle$ state making the total DOS at the Fermi energy small. In this way the double minimum structure in $\rho_{x x}$ can be explained.

For low carrier densities $N_{s}=3.0 \times 10^{11} \mathrm{~cm}^{-2}$ (see Fig. 3) the upper subband is just barely populated (Fig. 2) at $B=0$. For high magnetic fields the upper subband becomes depopulated at integer filling factors and therefore $v=4=v^{0}+v^{1}=4+0$. At high carrier densities $N_{s}=4.6 \times 10^{11} \mathrm{~cm}^{-2}$ the upper subband can gain carriers at the cost of the lower one and $v=4=2+2$ will be realized. This results in a quantum Hall state and $N_{s}^{0}=N_{s}^{1}$. Such a situation cannot be realized in a triangular shaped potential because there the carrier density in an upper subband $N_{s}^{1}$ will always be much lower than $N_{s}^{0}$. Consequently the upper subband will be depopulated at $v=4$. For intermediate carrier densities $N_{s}=3.8 \times 10^{11} \mathrm{~cm}^{-2}$ the influence of the magnetic field on the subband structure is not strong enough to either depopulate the upper subband or match the carrier densities of the two subbands. Two Landau levels originating from different subbands $|0,1\rangle$ and $|1,0\rangle$ can be degenerate at the Fermi energy and the quantum Hall state corresponding to $v=4$ is suppressed (see Fig. 3).

The question arises whether the degeneracy of Landau

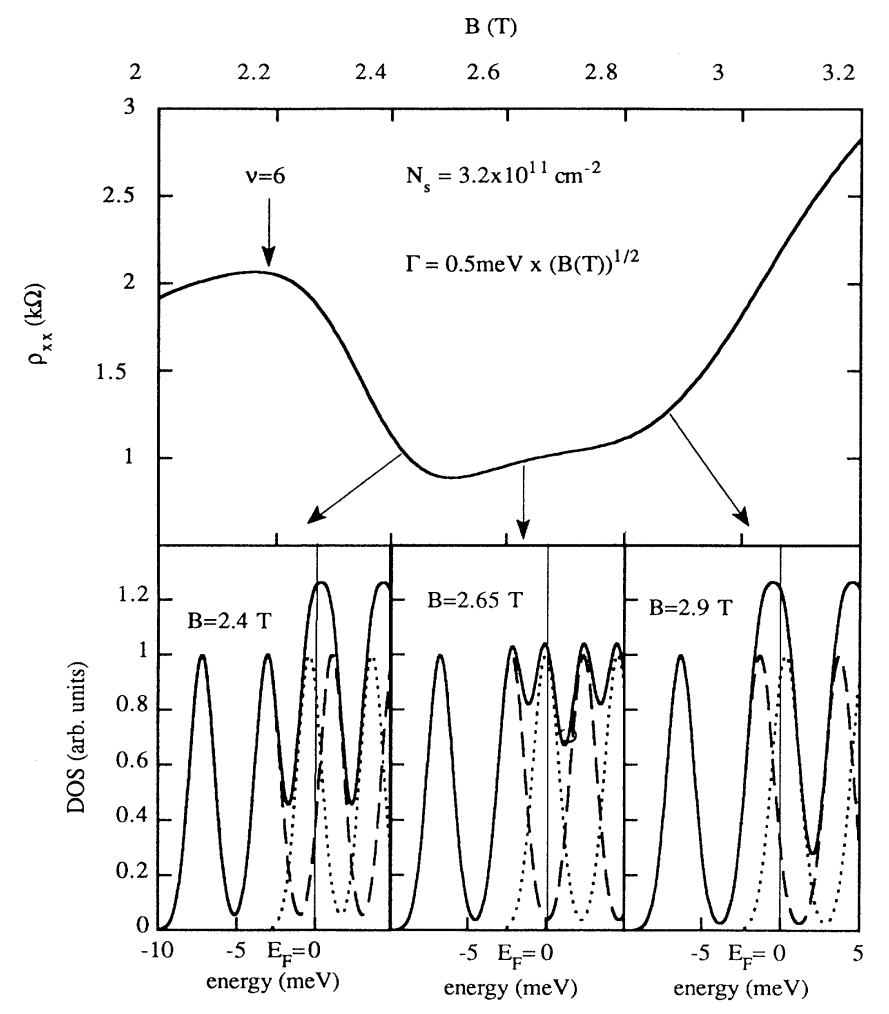

FIG. 5. Calculated magnetoresistance trace presented with an enlarged scale compared to Fig. 5. The lower part of the figure presents three situations for the density of states as marked by the arrows. The dashed line marks the DOS of the lower $E_{0}$ subband; the dotted line marks the DOS correspondingly of the $E_{1}$ subband, and the solid line indicates the total DOS. The thin solid vertical line represents the position of the Fermi energy $E_{F}=0$. 
levels can also lead to the suppression of quantum Hall states for samples with very high mobility at very low temperatures. We therefore calculate the magnetoresistance $\rho_{x x}$ for very narrow Landau levels $\Gamma[\mathrm{meV}]=0.005 \times(B[T])^{1 / 2}$ at $T=0$. The subband structure is calculated self-consistently in the presence of a magnetic field and $\rho_{x x}$ is then obtained as described before. Fig. 6(a) presents the results in an enlarged scale compared to Fig. 4(b). The features are much sharper due to the narrow Landau levels but at intermediate carrier densities $N_{s}=3.6 \times 10^{11} \mathrm{~cm}^{-2}$ the position of $\nu=4$ is situated in a maximum of $\rho_{x x}$. Thus there exists a carrier density even for high mobility samples at very low temperatures where Landau levels will be degenerate and the corresponding quantum Hall state is expected to be suppressed.

For a comparison we calculate the subband structure self-consistently for $B=0$ and modeled the Landau levels on top of these subbands. The effects of the magnetic field on the subband structure are intentionally neglected. The results for $\rho_{x x}$ are presented in Fig. 6(b). The narrow Landau level moves through the $\rho_{x x}$ trace leaving the $v=4$ minimum almost unchanged. We conclude that the influence of the magnetic field on the subband structure itself is especially important for samples with very high mobility. The Hartree interaction tends to pin Landau levels of different subbands together to avoid a jump of the Fermi energy from a Landau level from subband $i=0$ to another Landau level from subband $i=1$. The pinning of Landau levels has also been observed via optical spectroscopy in high-density $\operatorname{In}_{x} \mathrm{Ga}_{1-x} \mathrm{As}-\mathrm{InP}$ quantum wells. ${ }^{35}$

It is interesting to discuss which even integer quantum

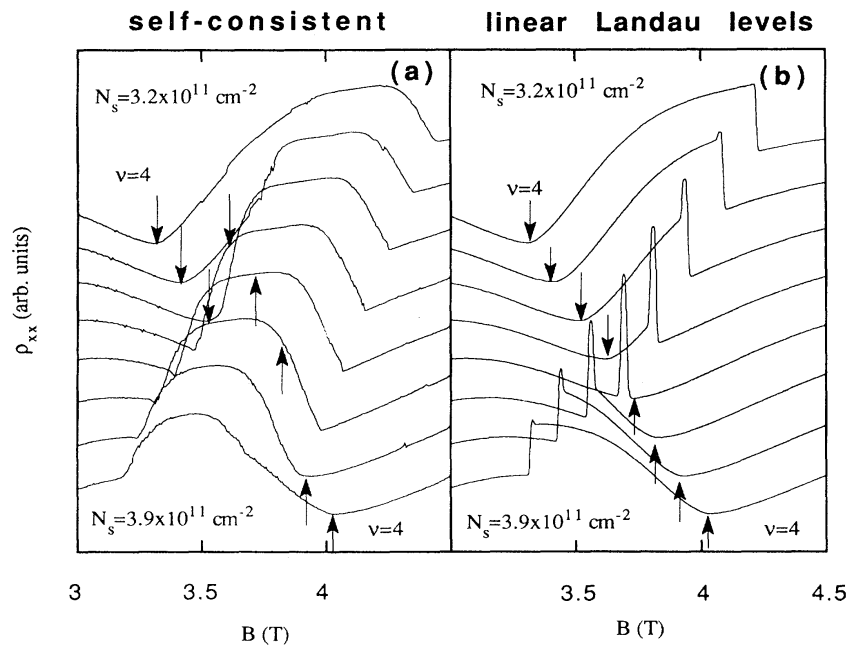

FIG. 6. (a) Self-consistent calculation of the magnetoresistance traces for very narrow Landau levels and $T=0$. The vertical arrows mark the position of filling factor $v=4$. It is important to note that for intermediate carrier density $N_{s}=3.5 \times 10^{11}$ $\mathrm{cm}^{-2}$ the position of the arrows sits in a maximum of $\rho_{x x}$. (b) Calculated magnetoresistance with a constant subband separation $E_{10}$, independent of magnetic field. The position of filling factor 4 as marked by the arrows is always in a minimum of $\rho_{x x}$.
Hall state can be suppressed. We again neglect spin splitting for this discussion. Filling factor $v=2$ is realized when only one Landau-level and therefore only one subband is occupied. This is true as long as the Landau-level width $\Gamma$ is smaller than the subband separation $E_{10}$ which is practically the case for all 2DEG's in GaAs heterostructures. Consequently the quantum Hall state corresponding to $v=2$, i.e., the quantum lines, can never be suppressed. The $v=4$ state can be suppressed as presented in Fig. 3 and discussed above. In our experiments we never observed a situation where the quantum Hall state corresponding to $v=6$ is suppressed. The width of the plateau may change, but the plateau is always well developed. The $v=8$ state can again be suppressed as discussed in Ref. 15. In general, an even integer quantum Hall state can be suppressed if odd filling factors can occur simultaneously in the respective subbands. This means that an even number has to be represented by the sum of two odd numbers, implying a similar ratio for the carrier densities in the two subbands, since the electrical confinement will always dominate the magnetic-fieldinduced energy changes. To suppress a quantum Hall state at even $v$, this filling factor has to be the sum of odd $v^{0}$ and odd $v^{1}$. The carrier density within a subband, in general, depends upon the magnetic field. To suppress a quantum Hall state two Landau levels have to be degenerate at the Fermi energy resulting in a large DOS at the Fermi energy. In such a "relaxed" situation the carrier densities in the two subbands have to be close to their $B=0$ values. Otherwise carriers could be easily distributed between the subbands due to the large DOS at $E_{F}$ without changing $E_{10}$ very much. We conclude that two conditions are important to observe the suppression of an even-integer quantum Hall state: (1) two odd filling factors $v^{0}$ and $v^{1}$ with $v^{0}+v^{1}=v$ have to be realized in the respective subband; (2) the ratio of the carrier densities at $B=0$ should be close to $N_{s}^{0} / N_{s}^{1} \cong v^{0} / v^{1}$. Furthermore $v^{0}$ and $v^{1}$ should be as close as possible to each other. Otherwise the magnetic field depopulates the higher subband and no quantum Hall state can be suppressed.

For example, the $v=4$ state is suppressed if $v=4=v^{0}+v^{1}=3+1$. With the same argument we find two possibilities for the $v=6$ state: $v=6=v^{0}+v^{1}=1+5$ and $v=6=v^{0}+v^{1}=3+3$. The latter condition does not exist because it implies $N_{s}^{0}=N_{s}^{1}$ and therefore a vanishing subband separation. The first condition $v=1+5$ corresponds to the already discussed situation. The carrier density in the upper subband $N_{s}^{1}$ is much smaller than $N_{s}^{0}$ and high magnetic fields will therefore depopulate the upper subband. We do not exclude that in very specially designed samples the $v=6$ state can also be suppressed, but we argue that it is much easier to suppress the $v=4$ state. Using the same reasoning we find two conditions to suppress the $v=8$ state: $v=8=v^{0}+v^{1}=5+3$ or $v=8=v^{0}+v^{1}=1+7$. The latter condition is again not favorable because high magnetic fields will depopulate the higher subband. The condition $v=5+3$ can however easily be realized leading to a suppression of the $v=8$ state. Figure 7 presents a schematic illustration for the suppression conditions of $v=4,8,12$. The Landau levels of the lower (upper) subband are indicated on the left- 


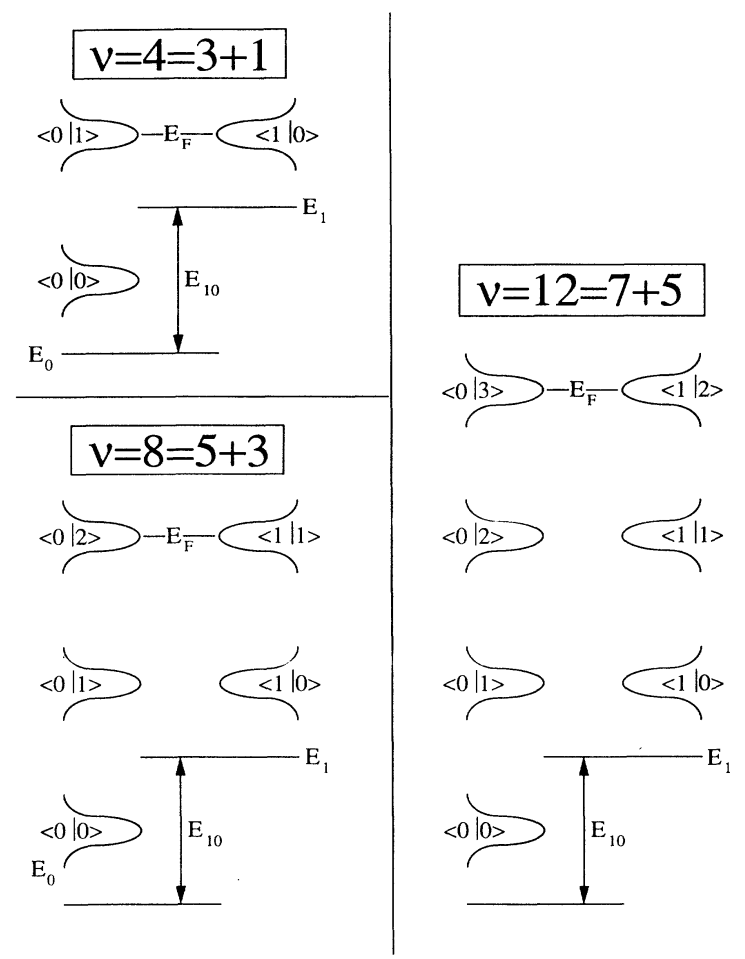

FIG. 7. Schematic illustration of three situations of the energy levels as observed in the experiments. On the left- (right-) hand side the Landau levels of the lower (upper) subband are indicated. Spin splitting is not considered in this picture.

(right-) hand side of the diagram. Along the same line we find for $v=10$ three possibilities: $v=10=9+1$, $v=10=7+3$, and $v=10=5+5$. The last case is excluded because it implies degeneracy of the electrical subband energies. The first two cases suggest again a situation where $N_{s}^{1} \ll<N_{s}^{0}$ which leads to a depopulation of the upper subband in a magnetic field. We note that we do not observe a suppression of the $v=10$ in the experiment in agreement with the presented argument. For filling factor $v=12$ again three possible scenarios are imaginable: $v=12=7+5, v=12=9+3$, and $v=12=11+1$. The last possibility is not realistic due to the abovepresented arguments, the first condition $v=12=7+5$ can be realized in the experiment. The situation $v=12=9+3$ implies $N_{s}^{0} \approx 3 N_{s}^{1}$ at $B=0$ and therefore coincides with the suppression of $v=4$ with $v=4=3+1$. Indeed we do observe a simultaneous suppression of the $v=4$ and 12 state. However the precise evaluation of the data for large filling factors is difficult because of the many overlapping Landau levels in this case. We conclude that there is a good understanding why and in which range of $N_{s}$ and $B$ a given even integer quantum Hall state can or cannot be suppressed.

\section{DEGENERACY OF SPIN SPLIT LEVELS}

So far the Zeeman spin splitting of Landau levels in a magnetic field has been neglected. Nevertheless it should be possible to study the situation where spin-split levels originating from different subbands are degenerate at the
Fermi energy. We restrict the following discussion to the intriguing behavior of the $v=3$ state. It is the best developed spin-split state in the presented experiment and theoretically it allows for a transparent discussion. Figure 8 presents a series of $\rho_{x x}$ data measured at low temperatures $T=0.45 \mathrm{~K}$ for a range of carrier densities where the suppression of the $v=4$ state occurs. For high carrier densities and $V g=-150 \mathrm{mV}$ (lowest-lying curve) pronounced minima are observed for $v=2,3,4,5,6$ and so on. The uppermost curve with $V g=-390 \mathrm{mV}$ shows well-developed $\rho_{x x}$ minima at $v=1,2,4,6$ and a weak minimum at $v=3$. The curves for intermediate carrier densities $-390 \mathrm{mV}<V g<-150 \mathrm{mV}$ indicate many competing minima for $v=3$ and 4 . The minima do not lie on the precise position of the respective filling factor.

Figure 9 presents the corresponding $\rho_{x y}$ data for the same range of gate voltages. Well-developed quantum Hall plateaus are observed for $v=1$ and 2. For filling factors $v=3$ and 4 the quantum Hall plateaus display a variety of features. Especially interesting is the case where $\rho_{x y}$ drops for increasing $B$ and then rises again. This situation cannot be observed at high temperatures where spin splitting is not resolved. The fact that strong spin splitting can be observed in the magnetoresistance $\rho_{x x}$ of GaAs heterostructures ${ }^{36}$ is based on the exchange enhanced $g$ factor. ${ }^{37}$ For odd filling factors a filled Landau level at the Fermi energy is fully spin polarized because all occupied states are filled with electrons of the same spin orientation. According to Ando and Uemura (Ref. 37) the exchange enhanced $g$ factor can be written as

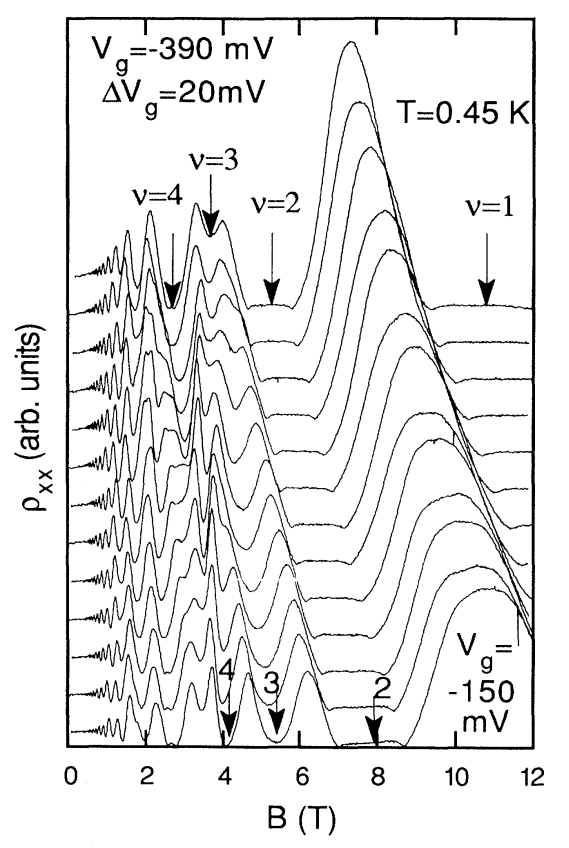

FIG. 8. Experimental magnetoresistance traces for a series of gate voltages at low temperatures $T=0.45 \mathrm{~K}$. The arrows mark the position of filling factors. The curves are vertically offset for clarity. An interesting double minimum structure occurs for intermediate carrier densities around filling factor $v=3$. 


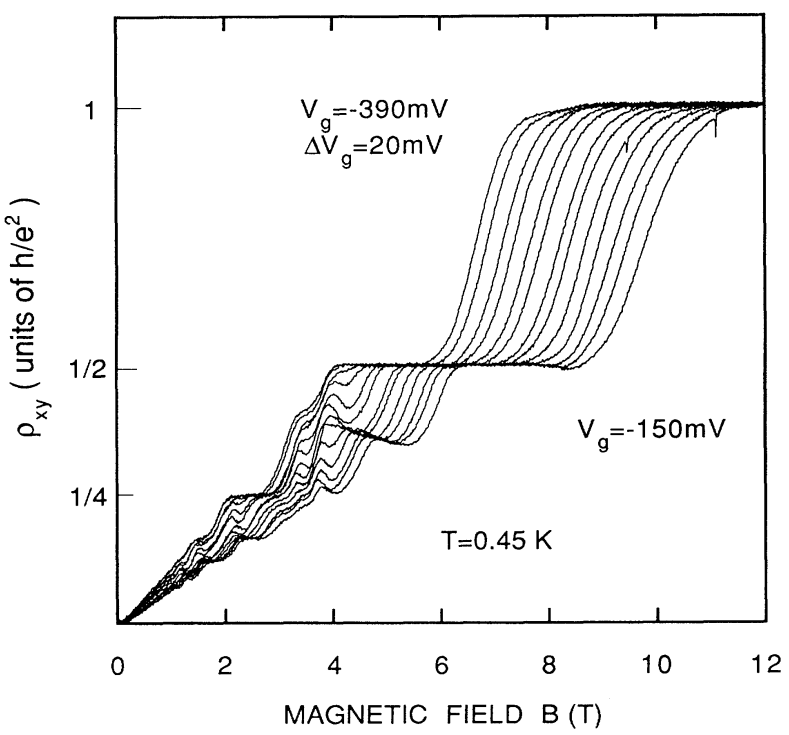

FIG. 9. Hall effect $\rho_{x y}$ for the same range of carrier densities as in Fig. 10. Overshoots of the Hall plateau occur for $v=3$ and 4.

$$
g^{*}=g\left(1+\frac{1}{g \mu B} \sum_{N^{\prime}} \sum_{q} \frac{V(q)}{\varepsilon(q, 0)}\left(n_{N^{\prime} \uparrow}-n_{N^{\prime} \downarrow}\right) J_{N N^{\prime}}^{2}(q)\right) .
$$

Here $V(q)$ is the effective two-dimensional Coulomb potential of the electrons, $\varepsilon(q, 0)$ the dielectric function, and $J_{N N^{\prime}}^{2}(q)$ the matrix element of the interaction between electrons in Landau levels $N$ and $N^{\prime}$. The population difference of the two spin-split levels is denoted by $n_{N \uparrow}-n_{N \downarrow}$. The value of $n \downarrow(n \uparrow)$ is the number of all spin down (up) electrons in all occupied Landau levels of subband $i$. In a simplified form this can be written as

$$
g^{*}=g\left[1+\alpha_{\mathrm{ex}}(n \uparrow-n \downarrow)\right],
$$

where $\alpha_{\text {ex }}$ now depends on the exchange interaction and also on the subband quantum number $i$ : The important consequence for our experiment is that the energy separation of spin-split Landau levels depends on the filling of these levels $(n \uparrow, n \downarrow)$ as well as on the subband quantum number. We will therefore denote the bare $g$ factor in subband $i$ by $g_{i}$, the enhanced $g$ factor by $g_{i}{ }^{*}$. To realize filling factor $v=3$ there are, in principle, two possibilities that are indicated schematically in Fig. 10. If the $|i=0, n=1\rangle$ and $|1,0\rangle$ levels are degenerate the exchange enhancement may lead to a situation where the spin down $(\downarrow)$ level of the $|0,1\rangle$ state drops in energy and $v=3$ is realized by $v=v^{0}+v^{1}=3+0$. Alternatively the enhanced spin splitting may occur in the higher-lying subband resulting in $v=3=2+1$. These two cases differ dramatically in the carrier densities they assign to the two electrical subbands. The exchange enhancement in the second case will be larger because the upper subband is completely spin polarized $(n \uparrow=0)$ whereas the situation as sketched on the left hand side of Fig. 10 occurs due to the spin splitting of the $n=1$ Landau level in the lower subband. The enhanced $g$ factor $g_{0}^{*}$ will be smaller because of the spin-unpolarized Landau level $n=0$ below
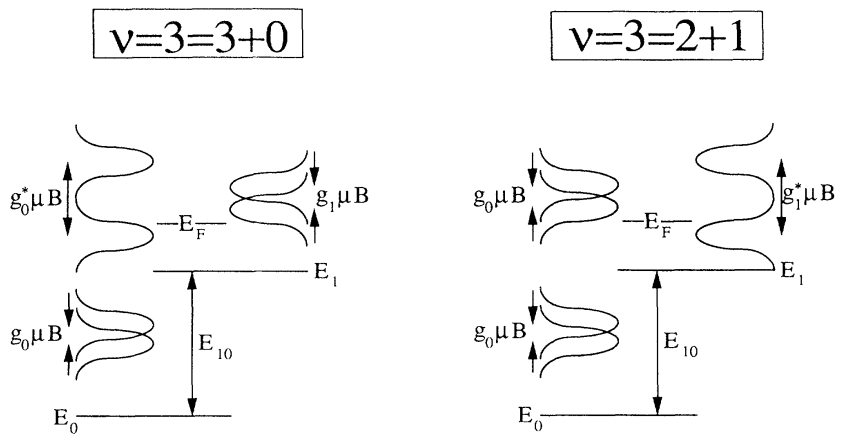

FIG. 10. Schematic representation of two situations that result in three occupied Landau levels. The bare $g$ factor of the system is marked by $g_{0}$ and $g_{1}$, the enhanced $g$ factor of the upper and lower subband by $g_{0}^{*}$ and $g_{1}^{*}$, respectively.

the spin-polarized state in the $n=1$ Landau level. We conclude that the interesting double minimum structure in Fig. 8 around $v=3$ occurs because the enhancement of the $g$ factor is different in the two subbands.

This effect will also influence the suppression behavior of the $v=4$ state, since, for resolved spin slitting, $v=4$ can be expressed as $v=4+0$ or $v=3+1$. Again these two situations result in a completely different ratio of carriers in the two subbands (4:0 vs 3:1). Overshoots of quantum Hall plateaus have also been observed for spinsplit states ${ }^{38}$ in GaAs heterostructures with one occupied subband. The temperature and geometry dependence of this effect implies an explanation that relies on the magnetic-field-induced decoupling of the two edge states associated with the topmost spin-split Landau level. In our experiment here on a parabolic quantum well with two occupied subbands we observe similar effects around $v=3$ as presented in Fig. 9. In addition to the explanation presented in Ref. 38, we think that the subband dependent enhancement of the $g$ factor as sketched in Fig. 10 further influences the detailed shape of a quantum Hall plateau. In a system with two occupied subbands the spin splitting of Landau levels can also account for features of even integer quantum Hall states.

\section{TILTED FIELD EXPERIMENTS: MANIFESTATION OF RESONANT-SUBBAND-LANDAU-LEVEL COUPLING}

The magnetotransport data presented so far in this paper were obtained for magnetic fields oriented perpendicular to the plane of the sample. In this case the Hamiltonian can be separated into two terms, one describing the in-plane motion of the carriers and the other the electrostatic confinement in the perpendicular direction. ${ }^{39}$ The energy-level structure then consists of an equidistant ladder of Landau levels on each electrical subband. The Landau levels associated with different subbands may cross each other without any interaction because the Hamiltonian is separable. In this section we concentrate on the case of tilted magnetic fields where the Hamiltonian can no longer be separated. Consequently Landau levels belonging to different subbands are coupled. This coupling has been observed by far-infrared spectrosco- 
py $^{4-9}$ but is difficult to tackle in a transport experiment. Figure 11(a) presents magnetoresistance data for a series of tilt angles $\alpha$ between the sample normal and the orientation of the magnetic field. The curves are vertically offset for clarity. The measurement temperature is $T=2.2 \mathrm{~K}$ so that spin effects are only weakly pronounced. We will consequently neglect the spin splitting of the Landau levels in the following discussion of RSLC. The gate voltage in Fig. 11(a) is chosen such that the $\rho_{x x}$ minimum and with it the corresponding quantum Hall state for $v=4$ is suppressed at $\alpha=0$. The weak minimum between the arrows marking $v=4$ and 2 corresponds to the $v=3$ state. For increasing tilt angle the minimum for $\nu=4$ starts to reappear and is finally fully recovered for $\alpha=35.9^{\circ}$. At the same time the neighboring minimum for the $v=6$ suffers the opposite fate. For perpendicular magnetic fields, $\alpha=0$, the $v=6$ state is well pronounced. As the tilt angle is increased the minimum for $v=6$ is completely suppressed at $\alpha=35.9^{\circ}$. The recovery of the $v=8$ state for tilted magnetic fields is described in detail in Ref. 15. The essential features of the magnetoresistance traces in Ref. 15 look very similar as in Fig. 11(a), which shows the recovery of the $v=4$ state. In order to understand this remarkable observation in more detail we have solved Schrödinger's and Poisson's equation for $B=0$. The results of Sec. III indicate that the electrical subband energies depend on a perpendicular $(\alpha=0)$ magnetic field. The magnitude of these effects is comparable to the typical width $(0.5 \mathrm{meV})$ of a Landau level. For tilted magnetic fields however, $\alpha \neq 0$, the level repulsion is the decisive quantity being larger than typical effects of the magnetic field on the subband structure itself. This justifies the use of a harmonic approximation for the evaluation of the level anticrossing even though the selfconsistent potential is no longer parabolic. We therefore

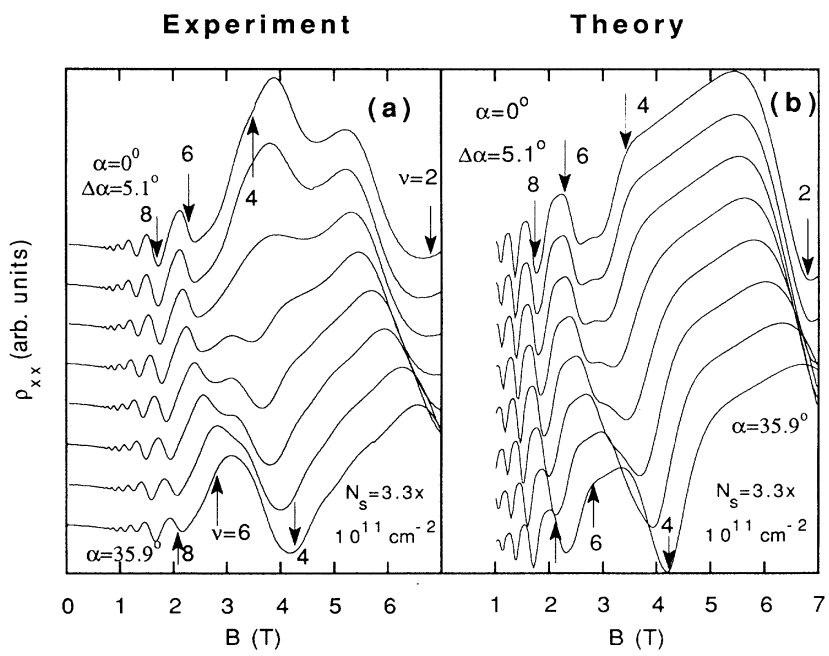

FIG. 11. (a) Experimental magnetoresistance $\rho_{x x}$ for a series of tilt angles $\alpha$ at $T=2 \mathrm{~K}$. The carrier density is fixed via a suitable gate voltage so that the minimum in $\rho_{x x}$ corresponding to filling factor $v=4$ is suppressed for $\alpha=0$. The curves are vertically offset for clarity. (b) Calculated magnetoresistance for the same parameter range as the experimental curves in Fig. 11(a). use the subband separation $E_{10}=E_{1}-E_{0}$ as obtained from the self-consistent calculation to model a parabolic potential with characteristic frequency $\hbar \omega_{0}=E_{10}$. The case of a parabolic potential in a tilted magnetic field can be solved analytically ${ }^{40,41}$ and the level repulsion can be obtained. With $\omega_{c}=e B / m^{*}$ being the cyclotron frequency the Hamiltonian can be rewritten after a rotation of the coordinate system and one obtains two decoupled harmonic oscillators with frequencies

$$
\begin{aligned}
\omega_{1 / 2}^{2}= & \frac{1}{2}\left(\omega_{c}^{2}+\omega_{0}^{2}\right) \\
& \pm \frac{1}{2}\left[\omega_{c}^{4}+\omega_{0}^{4}+2 \omega_{0}^{2} \omega_{c}^{2}\left(\sin ^{2} \alpha-\cos ^{2} \alpha\right)\right]^{1 / 2} .
\end{aligned}
$$

The energy spectrum is then given by

$$
E_{k m}=\hbar \omega_{1}\left(k+\frac{1}{2}\right)+\hbar \omega_{2}\left(m+\frac{1}{2}\right) .
$$

According to the procedure as described in Sec. III the magnetoresistance $\rho_{x x}$ is calculated. This calculation is based on the subband quantum number $i$ and the Landau-level quantum number $n$. For tilted magnetic fields hybrid electric magnetic subbands are formed and $i$ and $n$ are no longer good quantum numbers. Consequently the carrier density of a given subband is not a well-defined quantity anymore, especially in the vicinity of a resonant level anticrossing. Nevertheless we associate the new quantum number $k$ and $m$ with the old ones $i$ and $n$ even for $\alpha \neq 0$ as far as the transport calculations are concerned, to accomplish a transparent picture of the experimental situation. A minimum in $\rho_{x x}$ will always correspond to a position of the Fermi energy between two energy levels. The position of the calculated $\rho_{x x}$ minima will therefore not suffer from our assumption. The heights and positions of the maxima are more problematic, since they depend more sensitively on the subband carrier densities and the quantum numbers. The results of this calculation are plotted in Fig. 11(b) in the same way as the experimental data in Fig. 11(a) is presented. The reappearance of the $v=4$ minimum for increasing tilt angle as well as the suppression of the $v=6$ minimum are very well described by this calculation justifying the approximations that were made. The detailed shape of the oscillations especially in the vicinity of the maxima in $\rho_{x x}$ differs slightly between experiment and theory.

Figure 12 presents the calculated level structure for the typical tilt angles. For $\alpha=0$ the levels are decoupled and the $|0,1\rangle$ and $|1,0\rangle$ states are degenerate at the Fermi energy resulting in the suppression of the $v=4$ state. For increasing tilt angle $\alpha=15.4^{\circ}$ these levels start to repel each other showing a well-defined energy gap at $\alpha=35.9^{\circ}$ for $v=4$. Simultaneously the jump of the Fermi level at $v=6$ gets smeared out for increasing tilt angle in agreement with the suppression of the $v=6$ state as observed in the experiment [Fig. 11(a)]. The $|0,2\rangle$ and $|1,0\rangle$ state will repel each other at higher tilt angles leading then to a recovery of the $v=6$ state. We conclude that the level repulsion caused by the tilted magnetic field strongly manifests in the $\rho_{x x}$ spectra and is well explained by the calculation. Transport experiments can therefore be used to determine the single-particle subband separation of a 2DEG. In analogy to optical experiments the results of 


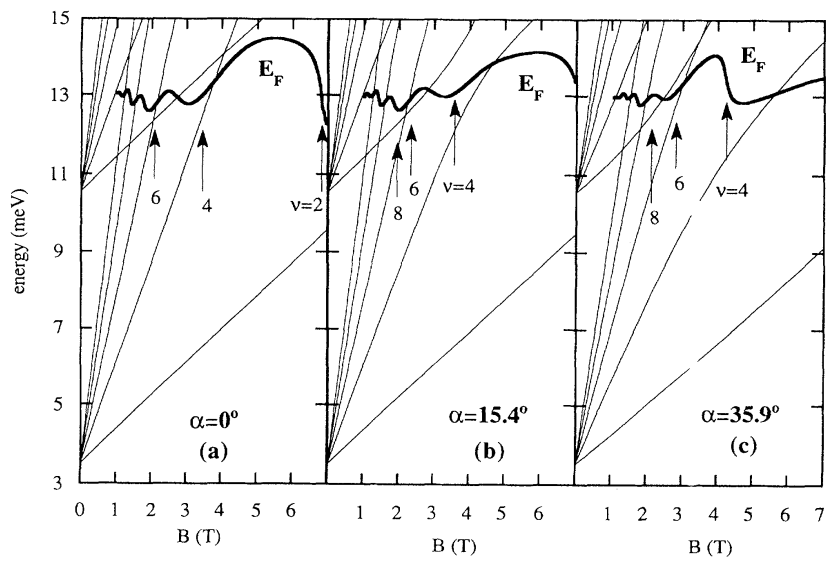

FIG. 12. Calculated energy-level structure for the same carrier density $N_{s}=3.3 \times 10^{11} \mathrm{~cm}^{-2}$ as in Fig. 11 . For increasing tilt angle the energy gap at the Fermi energy for $v=4$ opens up resulting in the recovery of the $v=4$ minimum as depicted in Fig. 11.

the transport experiments in Fig. 11(a) may be interpreted as an exchange of oscillator strength between the $v=4$ and the $v=6$ state.

Figure 13 presents a comparison of three experimentally determined values for the subband separation $E_{10}$ and the value of $E_{10}$ from a self-consistent calculation. According to the arguments as presented in Sec. III only the suppression of the $v=4,8$, and 12 state can clearly be observed in the experiment. From Fig. 11(a), which starts from a suppressed $v=4$ state at $\alpha=0$, the angle $\alpha$ and magnetic field can be deduced at which this state is recovered. With $B \cos \alpha=E_{10}$ we can extract the subband separation for this given carrier density $N_{s}=3.3 \times 10^{11} \mathrm{~cm}^{-2}$. Accordingly we find values for

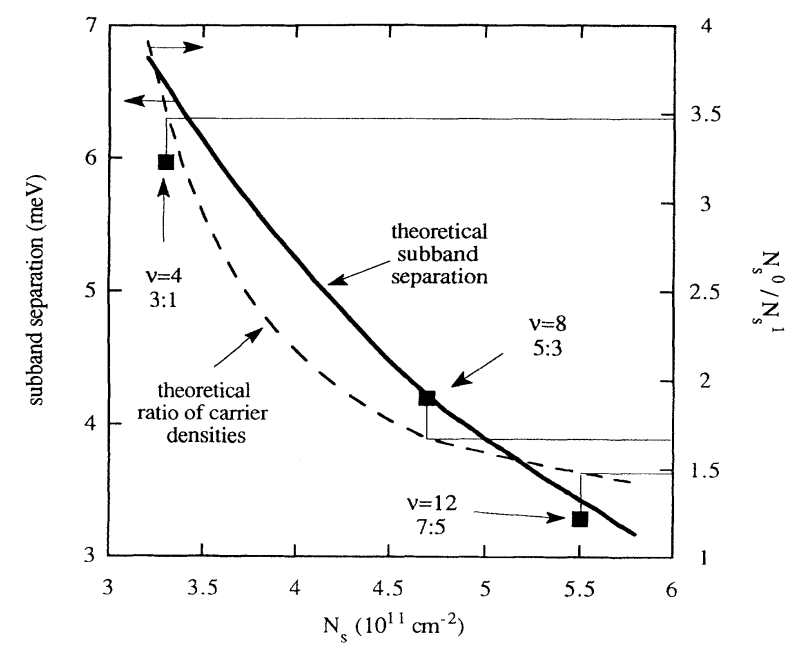

FIG. 13. The squares indicate the experimental results for the subband separation $E_{10}$ as a function of carrier density as obtained from RSLC. The thick solid line is the result of a selfconsistent calculation. The dashed line presents the calculated ratio of the carrier densities in the two subbands at $B=0$. The thin solid lines are guides to the eye as described in the text.
$E_{10}\left(N_{s}\right)$ for the suppressed $v=8$ state (Ref. 24) and the $v=12$ state as marked by the solid squares in Fig. 13. The full line is the result of the self-consistent calculation for $B=0$ in good agreement with the experimental results. The situation to suppress the $v=4$ state at $\alpha=0$ is most favorable, if the ratio of the carrier densities in the two subbands at $B=0$ is $N_{s}^{0}: N_{s}^{1} \approx 3: 1$ because then $v=4=3+1$ can be realized easily in a magnetic field. The dashed line in Fig. 13 marks the result of the selfconsistent calculation for the ratio of carrier densities $N_{s}^{0}: N_{s}^{1}$ as a function of total carrier density. The thin solid line starting from the full square corresponding to $v=4$ leads vertically until it crosses the dashed line and then moves over to the right-hand scale to end at a value that is not too far off from the desired $N_{s}^{0}: N_{s}^{1} \rightarrow 3: 1$. The same is done for the $v=8\left(N_{s}^{0}: N_{s}^{1} \rightarrow 5: 3\right)$ and $v=12\left(N_{s}^{0}: N_{s}^{1} \rightarrow 8: 5\right)$ state. In all cases the calculated ratio of the carrier densities agrees roughly with the desired value for the suppression of the respective state. We conclude that there is a qualitative understanding of the processes that lead to a suppression of quantum Hall states at $\alpha=0$ and their subsequent recovery for tilted magnetic fields.

\section{DEPOPULATION OF SUBBANDS VIA IN-PLANE FIELDS}

So far we have described transport techniques that allow the evaluation of single-particle energies from the analysis of Shubnikov-de Haas oscillations in tilted or perpendicular magnetic fields. A complementary technique uses in-plane fields to depopulate subbands via the diamagnetic shift. This technique has been applied by many groups. ${ }^{42-50}$ The band structure for in-plane magnetic fields is fundamentally different compared to situations with $\alpha \neq 90^{\circ}$. For $0 \leq \alpha<90^{\circ}$ there is always a component of the magnetic field perpendicular to the plane of the sample that allows for a plane-wave type wave function of the electrons. This in turn leads to a degeneracy of the levels proportional to $B_{\perp}=\cos \alpha B$. The Shubnikov-de Haas oscillations, that rely on this degeneracy, can be observed up to tilt angles of $\alpha=89^{\circ}$ and more (see, for example, Refs. 27 and 48). For $\alpha=90^{\circ}$ the situation is fundamentally different and an additional type of magnetoresistance oscillation arises, the so-called diamagnetic Shubnikov-de Haas effect. ${ }^{42}$ Figure 14(a) presents results for two orientations of current flow, parallel $\left(\rho_{z z}\right)$ and perpendicular $\left(\rho_{x x}\right)$ to the magnetic field, that lies in the plane of the electron gas. The inset clarifies the orientation of current flow with respect to the magnetic-field direction. The voltage across the voltage probes of the Hall bars should be ideally zero in this geometry. From the finite voltage that we detect in the experiment we estimate the accuracy of the orientation to be $\alpha=90^{\circ} \pm 0.01^{\circ}$. The experiment in Fig. 14(a) clearly shows the influence of the orientation of current flow on the detailed shape of the magnetoresistance trace.

In the literature there is widespread agreement that the magnetoresistance oscillations for in-plane fields arise from the crossing of the diamagnetically shifted subband energies through the Fermi energy. The question, how- 


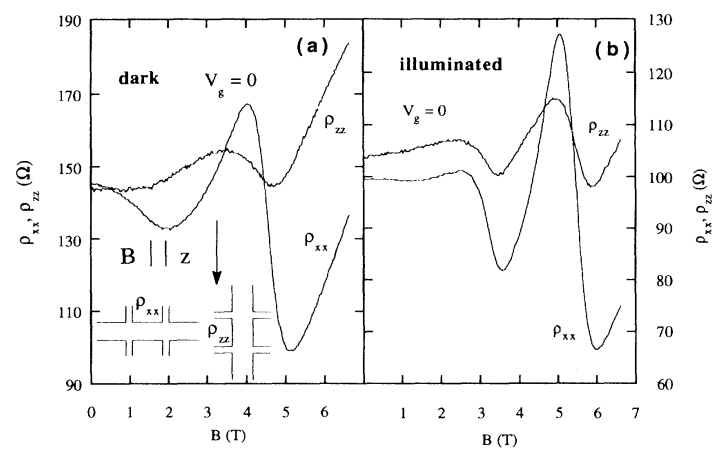

FIG. 14. (a) Magnetoresistance measured in the dark for inplane magnetic fields at $T=4.2 \mathrm{~K}$. The resistance for current flow parallel to the magnetic field is denoted by $\rho_{z z}$, for the perpendicular case by $\rho_{x x}$. The inset clarifies this situation. (b) Experimental magnetoresistance at $T=4.2 \mathrm{~K}$ after the sample has been strongly illuminated.

ever, at which point on the magnetoresistance trace (maximum, inflection point, minimum) the subband actually becomes depopulated is not satisfactorily answered so far. Furthermore, Fig. 14(a) indicates that the detailed shape of the magnetoresistance trace depends on the orientation of current flow. Most of the publications on the observation of the diamagnetic Shubnikov-de Haas effect do not mention the orientation of current flow in the experimental setup. Zrenner et al. ${ }^{46}$ investigated a Si $\delta$-doped layer in GaAs. They used a Corbino geometry and found nice agreement between the fields obtained from the maxima of $d \sigma / d B(B)$ traces and the depopulation fields from a self-consistent calculation. Kroeker and Merkt ${ }^{49}$ did research on a InSb metaloxide-semiconductor (MOS) structure. They found that the conductivity depends on the orientation of current flow. However the positions of the features caused by the magnetic depopulation of the subbands were independent on the orientation of current flow. The InSb-MOS structure resembles an asymmetric triangular-shaped potential for the electrons that accounts for the observed effects. In this case the electron trajectories depend on the orientation of current flow which therefore also influences the conductivity. Gwinn et al ${ }^{47}$ investigated $\rho_{z z}$ as a function of magnetic field. The positions of the maxima in the magnetoresistance $\rho_{z z}$ were in good agreement with the prediction of the depopulation fields according to a self-consistent calculation by Stopa and Das Sarma. ${ }^{51}$ Hopkins $^{50}$ investigated wide PQW's $(\approx 500 \mathrm{~nm})$ for both orientations of current flow and found pronounced anisotropies. The experimental results suggest, however, that the imperfections of the parabolic potential, that become more dominant the wider the well is, are crucial to explain the observed features.

The anisotropic band structure in the plane of the 2DEG arising from the in-plane magnetic field results in a magnetic-field-dependent effective mass perpendicular to the magnetic field of $m_{x}=m^{*} \omega^{2} / \omega_{0}^{2}$. Here $\omega_{0}$ is the harmonic oscillator frequency of the parabolic potential, $\omega_{c}=e B m^{*}$ the cyclotron frequency, and $\omega^{2}=\omega_{0}^{2}+\omega_{c}^{2}$.
This mass dispersion has been experimentally observed by Batke and $\mathrm{Tu} \mathrm{u}^{52}$ via grating coupler-induced plasmon resonances in GaAs heterostructures. The effective mass in the direction along the magnetic field ( $z$ direction) remains unchanged. In this case there is no effective Lorentz force because the directions of the drift velocity and the magnetic field are parallel.

To investigate the influence of the mobility of the anisotropic transport behavior the sample has been illuminated with a red light-emitting diode leading to an increase of the carrier density at $V_{g}=0$ from 5 to $N_{s}=7 \times 10^{11} \mathrm{~cm}^{-2}$ caused by the persistent photoconductivity effect. Simultaneously the mobility has dropped from $\mu=100.000 \mathrm{~cm}^{2} / \mathrm{V} \mathrm{s}$ down to $\mu=80.000 \mathrm{~cm}^{2} / \mathrm{V} \mathrm{s}$. Figure 14(b) presents data for $\rho_{x x}$ and $\rho_{z z}$ after the sample has been illuminated. There is still a pronounced anisotropy but the positions of the maxima and minima are now closer to each other. The smaller the mobility the lower is the scattering time and the more the electrons are scattered out of their drift velocity direction. They therefore experience the band structure in all directions. We conclude that the anisotropy in $\rho_{x x}$ and $\rho_{z z}$ is reduced for low-mobility samples in agreement with our experimental observations [Fig. 14(a) $\rightarrow$ Fig. 14(b)] and the results of Si- $\delta$ layers $^{46}$ and the InSb-MOS structures. ${ }^{49}$

On the theoretical side Tang and Butcher ${ }^{53,54}$ have calculated the transport coefficients assuming an energy independent DOS with $D=m^{*} \omega / \pi \hbar^{2} \omega_{0}$. Their model system was a PQW with $\hbar \omega_{0}=3 \mathrm{meV}$ and $N_{s}=5.8 \times 10^{11}$ $\mathrm{cm}^{-2}$. The mobility assumed in their calculation is about a factor of 20 below the mobility of our sample. In Ref. 53 they calculated $\rho_{x x}$ and in Ref. $54 \rho_{z z}$. We digitized their results and put it together in one diagram as depicted in Fig. 15. The main features are similar as observed in the experiment; however, the anisotropy is much stronger in the experimental data possibly caused by the lower electron mobility assumed in the calculation. Furthermore in the calculation the inflection point of the $\rho_{x x}$

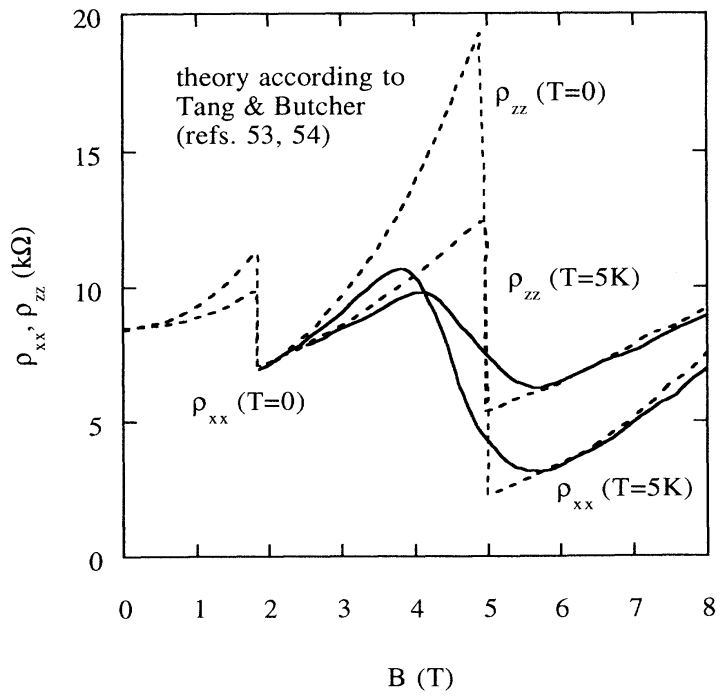

FIG. 15. Calculated magnetoresistance after Refs. 53 and 54. The dotted lines are for $T=0$, the solid line for $T=5 \mathrm{~K}$. 
trace lies at lower magnetic fields compared to the corresponding feature in the $\rho_{z z}$ trace. This is in contrast to the experimental findings [Fig. 14(a)].

Figure 16(a) presents a series of $\rho_{x x}$ measurements for various gate voltages. For high gate voltage, $V g=+200$ $\mathrm{mV}$, two oscillatory features are observed indicating at least three occupied subbands at $B=0$. For decreasing gate bias the low-field oscillation at around $B=2.5 \mathrm{~T}$ vanishes because the number of occupied subbands at $B=0$ is reduced to two. The oscillatory feature at around $B=5 \mathrm{~T}$ shifts successively to lower magnetic fields for decreasing gate voltage because of the changing subband structure. Figure 16(b) shows the corresponding spectra for current flow parallel to the magnetic-field orientation. The low-field oscillation vanishes in the same way as described in Fig. 16(a). The same correspondence is true for the high-field oscillation that results from the magnetic depopulation of the $E_{1}$ subband. The overall strength of the oscillations is however much weaker for current flow parallel to the magnetic field (Fig. 17) compared to the other current orientation.

For a more quantitative analysis we have extracted the magnetic-field positions of the minima, inflection points, and maxima of the magnetoresistance oscillations $\rho_{x x}$ and $\rho_{z z}$ as plotted in Fig. 17. For the low-field oscillation at around $B=2.5 \mathrm{~T}$ only the positions of the minima can be consistently evaluated. The data points for $\rho_{z z}$ lie consistently about $0.5 \mathrm{~T}$ below the ones of $\rho_{x x}$ as presented in Fig. 17. Following Ref. 53 a straightforward model is presented to allow a quantitative comparison of experiment and theory. The subband separation $E_{10}$ is calculated self-consistently for $B=0$. The potential is then approximated by a parabolic potential with eigenfrequency $\omega_{0}$ and $\hbar \omega_{0}=E_{10}$. Therefore $\omega_{0}=\omega_{0}\left(N_{s}\right)$ and the energy levels of the total confining potential are given by $E_{k}=\hbar \omega\left(k+\frac{1}{2}\right)$ with $\omega^{2}=\omega_{0}^{2}+\omega_{c}^{2}$ and $\omega_{c}=e B / m^{*} .{ }^{40,41}$ The depopulation of subband $n$ is then given by the condition $E_{F}=\hbar \omega\left(n+\frac{1}{2}\right)$. With the magnetic-fielddependent density of states

$$
D=m^{*} \omega / \pi \hbar^{2} \omega_{0}
$$

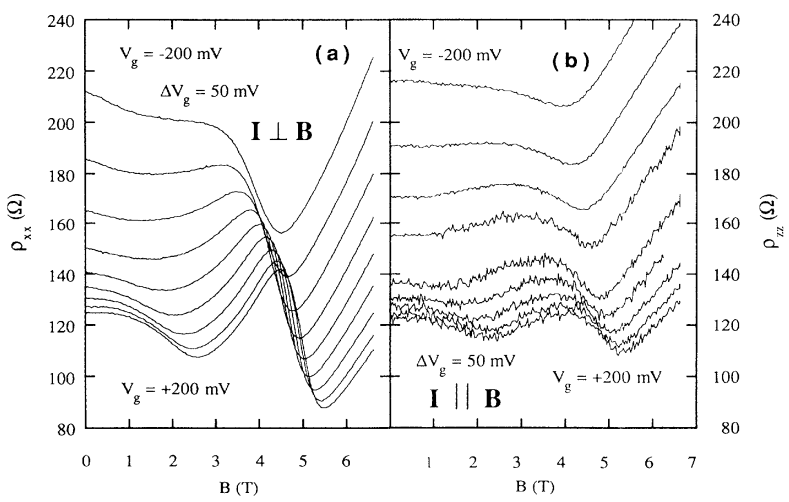

FIG. 16. (a) Experimental magnetoresistance for a series of gate voltages for current flow perpendicular to the magneticfield orientation at $T=4.2 \mathrm{~K}$. (b) Experimental magnetoresistance for current flow parallel to the magnetic-field orientation at $T=4.2 \mathrm{~K}$.

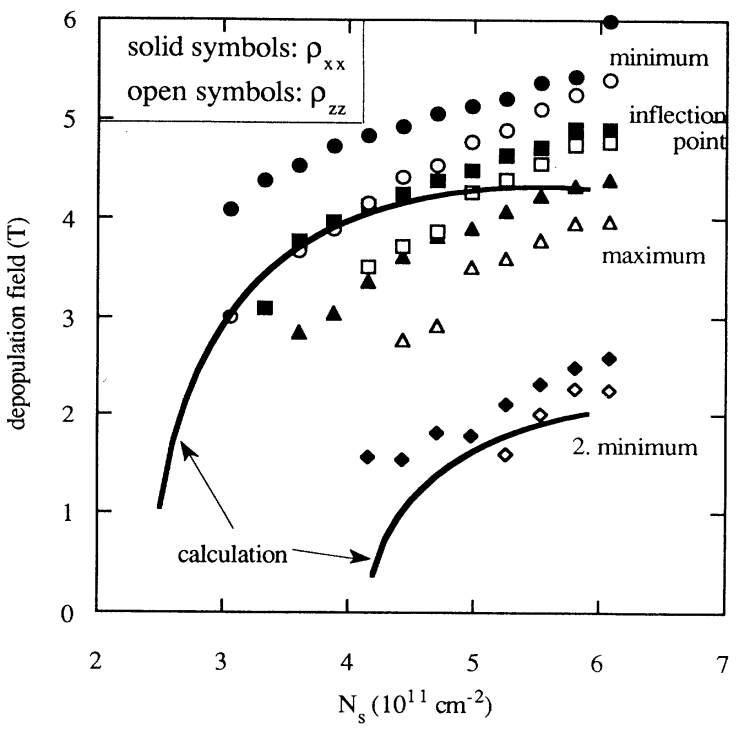

FIG. 17. Positions of the maxima, inflection points and minima along the magnetoresistance traces $\rho_{x x}$ (open symbols) and $\rho_{z z}$ (solid symbols) as a function of carrier density. The solid lines mark the result of the calculation as described in the text.

we get

$$
N_{s}=\frac{m^{*}}{\pi \hbar^{2}} \frac{\omega}{\omega_{0}} \sum_{k \leq n}\left[\hbar \omega\left(n+\frac{1}{2}\right)-\hbar \omega\left(k+\frac{1}{2}\right)\right] .
$$

This approximation is justified as long as the cyclotron diameter is larger than or comparable to the electronic width of the electron gas. For typical experimental conditions $\left(N_{s}=5 \times 10^{11} \mathrm{~cm}^{-2}, B=5 \mathrm{~T}\right)$ we obtain a classical cyclotron diameter of $47 \mathrm{~nm}$. This is larger than the effective width of the electron layer at the same carrier density which is about $40 \mathrm{~nm}$ (see Fig. 1). For lower values of the carrier density the cyclotron orbit as well as the width of the electron layer shrink. We conclude that the assumption of an energy-independent density of states is justified. Higher magnetic fields will result in a onedimensional-type density of states. ${ }^{46}$ The magnetic field $B_{n}$, where subband $n$ becomes depopulated, is then given by

$$
B_{n}=\frac{m^{*}}{\hbar e}\left[\left[\frac{2 N_{s}}{n(n+1)} \frac{\pi \hbar^{2}}{m^{*}}-\hbar \omega_{0}\right] \hbar \omega_{0}\right]^{1 / 2} .
$$

The self-consistency enters via $\omega_{0}=\omega_{0}\left(N_{s}\right)$ as calculated for $B=0$. The results of this calculation are depicted in Fig. 17 by solid lines. The main features of the experimental data are well explained by this model. Deviations occur for high magnetic fields, where the density of states becomes energy dependent ${ }^{46}$ as described above.

The energy separation $E_{23}$ is underestimated in the calculation because of the parabolic model. Intuitively Fig. 17 suggests, that the depopulation of the $E_{1}$ energy level occurs close to the inflection point of the $\rho_{x x}$ trace. The data further imply that one has to be careful with the assignment of a depopulation field from magnetoresistance measurements on high mobility structures. The detailed influence of the subband depopulation on the magne- 
toresistance traces is theoretically not well understood and requires future investigations.

\section{SUMMARY}

This paper presents a series of transport experiments that allow the evaluation of the single-particle subband separation in a parabolic quantum well. The special subband structure of a PQW results in the possible occupation of several electrical subbands even at high magnetic fields applied perpendicular to the plane of the electron gas. This fact can be used to study the case where Landau levels associated with different electrical subbands are degenerate at the Fermi energy. The experiments reveal the suppression of quantum Hall states which can be recovered by a lifting of the degeneracy. This can be achieved either by a change of the subband separation via a suitable gate voltage or by a tilt of the sample with respect to the magnetic-field axis that leads to a resonant level anticrossing. Especially interesting is the case, where for very low temperatures spin splitting of the Landau levels can be resolved. The magnetoresistance in this case is influenced by the subband dependent exchange enhancement, that leads to overshoots in the quantum Hall plateaus.

Finally an experiment is presented where, for in-plane magnetic fields, the magnetoresistance oscillations depend on whether the current flows parallel or perpendicular to the magnetic field. The positions of the oscillations are related to the magnetic depopulation of the electrical subbands. All experiments are accompanied by selfconsistent subband calculations that allow for a quantitative comparison with the experimental results.

\section{ACKNOWLEDGMENTS}

We like to thank J. P. Kotthaus, U. Merkt, and A. Zrenner for very enlightening discussion. Financial support by the Volkswagen Foundation and the Air Force is gratefully acknowledged.
${ }^{1}$ T. Ando, A. Fowler, and F. Stern, Rev. Mod. Phys. 54, 437 (1982).

${ }^{2}$ See, for example, A. Pinczuk and J. M. Worlock, Surf. Sci. 113, 69 (1982).

${ }^{3}$ A. Kamgar, R. Kneschaurek, G. Dorda, and J. F. Koch, Phys. Rev. Lett. 32, 1251 (1974).

${ }^{4}$ Z. Schlesinger, J. C. M. Hwang, and S. J. Allen, Jr., Phys. Rev. Lett. 50, 2098 (1983).

${ }^{5}$ G. L. J. A. Rikken, H. Sigg, C. J. G. M. Langerak, H. W. Myron, J. A. A. J. Perenboom, and G. Weimann, Phys. Rev. B 34, 5590 (1986).

${ }^{6}$ A. D. Wieck, J. C. Maan, U. Merkt, J. P. Kotthaus, K. Ploog, and G. Weimann, Phys. Rev. B 35, 4145 (1987).

${ }^{7}$ K. Ensslin, D. Heitmann, and K. Ploog, Phys. Rev. B 39, 10879 (1989).

${ }^{8}$ K. Karrai, H. D. Drew, H. W. Lee, and M. Shayegan, Phys. Rev. B 39, 10426 (1989).

${ }^{9}$ A. Wixforth, M. Sundaram, K. Ensslin, J. H. English, and A. C. Gossard, Surf. Sci. 267, 523 (1992).

${ }^{10}$ W. P. Chen, Y. J. Chen, and E. Burstein, Surf. Sci. 58, 263 (1976).

${ }^{11}$ S. J. Allen Jr., D. C. Tsui, and B. Vinter, Solid State Commun. 20, 425 (1976).

${ }^{12}$ L. Brey, N. F. Johnson, and B. I. Halperin, Phys. Rev. B 40, 10647 (1989).

${ }^{13}$ K. Karrai, X. Ying, H. D. Drew, and M. Shayegan, Phys. Rev. B 40, 12020 (1989).

${ }^{14}$ K. Ensslin, M. Sundaram, A. Wixforth, G. H. English, and A. C. Gossard, Phys. Rev. B 43, 9988 (1991).

${ }^{15}$ K. Ensslin, C. Pistitsch, A. Wixforth, M. Sundaram, P. F. Hopkins, and A. C. Gossard, Phys. Rev. B 45, 11407 (1992).

${ }^{16}$ M. Sundaram, A. C. Gossard, J. H. English, and R. M. Westervelt, Superlatt. Microstruct. 4, 683 (1988).

${ }^{17}$ A. Wixforth, M. Sundaram, K. Ensslin, J. H. English, and A. C. Gossard, Appl. Phys. Lett. 56, 454 (1990).

${ }^{18}$ H. L. Störmer, A. C. Gossard, and W. Wiegmann, Solid State Commun. 41, 707 (1982).

${ }^{19}$ J. J. Harris, D. E. Lacklinson, C. T. Foxon, F. M. Selten, A. M. Suckling, R. J. Nicholas, and K. W. Barnham, Semicond. Sci. Technol. 2, 783 (1987).
${ }^{20}$ T. P. Smith, II and F. F. Fang, Phys. Rev. B 37, 4303 (1988).

${ }^{21}$ F. F. Fang, T. P. Smith, III, and S. L. Wright, Surf. Sci. 196, 30 (1988).

${ }^{22}$ A. M. Kreschuk, M. Yu. Martisov, T. A. Polyanskaya, I. G. Savel'ev, I. I. Saidashev, A. Ya. Shik, and Yu. V. Shmartsev, Solid State Commun. 65, 1189 (1988).

${ }^{23}$ H. van Houten, J. G. Williamson, M. E. I. Broekaart, C. T. Foxon, and J. J. Harris, Phys. Rev. B 37, 2756 (1988).

${ }^{24}$ R. Fletcher, E. Zaremba, M. D'Iorio, C. T. Foxon, and J. J. Harris, Phys. Rev. B 38, 7866 (1988).

${ }^{25}$ T. P. Smith, III, F. F. Fang, U. Meirav, and M. Heiblum, Phys. Rev. B 38, 12744 (1988).

${ }^{26}$ K. Ensslin, D. Heitmann, and K. Ploog, Phys. Rev. B 37, 10150 (1988).

${ }^{27}$ R. M. Kusters, Ph.D. thesis, University of Nijmegeen, 1990.

${ }^{28}$ K. Hirakawa and H. Sakaki, Phys. Rev. B 33, 8291 (1986).

${ }^{29}$ R. R. Gerhardts, Surf. Sci. 58, 227 (1976).

${ }^{30}$ D. Weiss, V. Moser, V. Gudmundsson, R. R. Gerhardts, and K. von Klitzing, Solid State Commun. 62, 89 (1987).

${ }^{31}$ K. Ensslin, D. Heitmann, R. R. Gerhardts, and K. Ploog, Phys. Rev. B 39, 12993 (1989).

${ }^{32}$ See, for example, E. Gornik, R. Lassnig, G. Strasser, H. L. Störmer, A. C. Gossard, and W. Wiegmann, Phys. Rev. Lett. 54, 1820 (1985).

${ }^{33}$ G. Gobsch, D. Schulze, and G. Paasch, Phys. Rev. B 38, 10943 (1988).

${ }^{34}$ Y. Guldner, J. P. Vieren, M. Voos, F. Delahaye, D. Dominguez, J. P. Hirtz, and M. Razeghi, Phys. Rev. B 33, 3990 (1986).

${ }^{35}$ D. G. Hayes, M. S. Skolnick, D. M. Whittacker, P. E. Simmonds, L. L. Taylor, S. J. Bass, and L. Eaves, Phys. Rev. B 44, 3436 (1991).

${ }^{36}$ See, for example, R. J. Nicholas, R. J. Haug, K. von Klitzing, and G. Weimann, Phys. Rev. B 37, 1294 (1988).

${ }^{37}$ T. Ando and Y. Uemura, J. Phys. Soc. Jpn. 36, 959 (1974).

${ }^{38}$ See C. A. Richter, R. G. Wheeler, and R. N. Sacks, Surf. Sci. (to be published), and references therein.

${ }^{39}$ T. Ando, Phys. Rev. B 19, 2106 (1979).

${ }^{40}$ J. C. Maan, in Two-Dimensional Systems, Heterostructures and Superlattices, edited by G. Bauer, F. Kuchar, and H. Heinrich 
(Springer-Verlag, Berlin, 1984), p. 183.

${ }^{41}$ R. Merlin, Solid State Commun. 84, 99 (1987).

${ }^{42}$ W. Beinvogl, A. Kamgar, and F. Koch, Phys. Rev. B 14, 42274 (1976).

${ }^{43}$ Th. Englert, J. C. Maan, D. C. Tsui, and A. C. Gossard, Solid State Commun. 45, 989 (1983).

${ }^{44}$ H. Reisinger and F. Koch, Surf. Sci. 170, 397 (1986).

${ }^{45}$ D. J. Newson, K. F. Berggren, M. Pepper, H. W. Myron, G. J. Davies, and E. Scott, J. Phys. C 19, L403 (1986).

${ }^{46}$ A. Zrenner, H. Reisinger, F. Koch, K. Ploog, and J. C. Maan, Phys. Rev. B 33, 5067 (1986).

${ }^{47}$ E. G. Gwinn, R. M. Westervelt, P. F. Hopkins, A. J. Rimberg,
M. Sundaram, and A. C. Gossard, Phys. Rev. B 39, 6260 (1989).

${ }^{48}$ M. Shayegan, T. Sajoto, J. Jo, M. Santos, and H. D. Drew, Phys. Rev. B 40, 3476 (1989).

${ }^{49}$ M. Kroeker and U. Merkt, Solid State Commun. 69, 699 (1989).

50P. F. Hopkins, Ph.D. thesis, Harvard University, 1990.

${ }^{51}$ M. P. Stopa and S. DasSarma, Phys. Rev. B 40, 10048 (1989).

${ }^{52}$ E. Batke and C. W. Tu, Phys. Rev. B 34, 3027 (1986).

${ }^{53}$ H. Tang and B. N. Butcher, J. Phys. C 21, 3313 (1988).

${ }^{54}$ H. Tang and B. N. Butcher, J. Phys. C 21, 3959 (1988). 